\title{
Use of Dysmorphology for Subgroup Classification on Autism Spectrum Disorder in Chinese Children
}

\author{
Virginia CN Wong \\ Cecilia KY Fung \\ Polly TY Wong
}

Professor Virginia CN Wong, MBBS, DCH (London, Glasgow), FRCP (London, Edinburgh), FHKAM (Paed), FHKCPaed, FRCPCH, Division of Child Neurology/Developmental Paediatrics / Neurohabilitation, Division of

Neurodevelopmental Paediatrics, Department of Pediatrics \& Adolescent Medicine, The University of Hong Kong, Hong Kong, China.

Cecilia KY Fung (BSc, MMedSc) The University of Hong Kong

Polly TY Wong (BSc, MPhil) The University of Hong Kong

Running Head: Subgroup Classification for ASD 
Data from 1261 Chinese Autistic Spectrum Disorder (ASD) patients were evaluated and categorized into dysmorphic (10.79\%) and non-dysmorphic groups (89.21\%) upon physical examination by the presence of dysmorphic features. Abnormal MRI/ CT result, IQ scores and epilepsy were significantly associated with the dysmorphic group of ASD children. However, gender, EEG abnormality and family history and recurrence of ASD were not found to be significantly different between group statuses. It is suggested that results collected from the Chinese population generally resembles that found in the Caucasians with ethnical differences still present. Current study supports the result shown in Miles' study (Miles et al. 2005), in which heterogeneity subtypes of autism of different genetic origins which could be distinguished by presence of dysmorphic features on the patients.

125 words

Keywords: Autism Spectrum Disorder; Dysmorphology; Subgroup Classification

Correspondence for this article should be addressed to Professor Virginia CN Wong, Department of Pediatrics \& Adolescent Medicine, The University of Hong Kong, Hong Kong, China.;

Fax: (852)-2855-1523; Email: vcnwong@hkucc.hku.hk 
Use of Dysmorphology for Subgroup Classification on Autism Spectrum Disorder in Chinese Children

Running Head: Subgroup Classification for ASD 
Data from 1261 Chinese Autistic Spectrum Disorder (ASD) patients were evaluated and categorized into dysmorphic (10.79\%) and non-dysmorphic groups (89.21\%) upon physical examination by the presence of dysmorphic features. Abnormal MRI/ CT result, IQ scores and epilepsy were significantly associated with the dysmorphic group of ASD children. However, gender, EEG abnormality and family history and recurrence of ASD were not found to be significantly different between group statuses. It is suggested that results collected from the Chinese population generally resembles that found in the Caucasians with ethnical differences still present. Current study supports the result shown in Miles' study (Miles et al. 2005), in which heterogeneity subtypes of autism of different genetic origins which could be distinguished by presence of dysmorphic features on the patients.

125 words

Keywords: Autism Spectrum Disorder; Dysmorphology; Subgroup Classification 


\section{Introduction}

Autism Spectrum Disorder (hereafter referred interchangeably as ASD or autism) is a neurodevelopmental disorder that originates from both genetic and environmental factors. Diagnostic features include abnormalities in language and communication; reciprocal social interactions presented with restricted, repetitive and stereotyped patterns of behaviors, interests and activities. ASD is usually evident within the first three years of age and affecting individual function in many social situations. The most recent figure from the Centres of Disease Control and Protection (CDC) estimates 1 in 88 children (11.3 per 1,000) with ASD in the United States (ADDM, 2012). In Study Country, Wong and Hui (2008) estimated the incidence of ASD as 5.49 per 10000 with a prevalence of 16.1 per 10,000 for children less than 15 years old with a male-to-female ratio of 6.58:1.

Recently, minor physical anomalies and dysmorphic features have been reported in meta-analysis for the Caucasian ASD populations (Ozgen et al. 2010). Specific anomalies and dysmorphic features previously reported include abnormal head circumference, abnormal cephalic index, abnormal palate, abnormal rotation of ears, abnormal interpupillary distances, deep-set nails, tapered fingers, wide nasal bridge, lower anterior hair line and thin upper lips (Rodier et al. 1997; Fombonne et al. 1999; Miles et al. 2008; Tripi et al. 2008). Marcocephaly was reported in $16.7 \%$ of the ASD study population by Fombonne et al. (1999); and study group by Tripi et al. (2008) further reported abnormal head circumference, abnormal cephalic index and abnormal palate. Posterior rotation of the external ears, small feet and normal-to-large hands 
and reduction of interpupillary distances were found in a study by Rodier et al. (1997).

In the study by Miles et al. (2005), children with autism were classified into two subgroups; 'complex autism' and 'essential autism'. It was found that a proportion of children with autism suffered with abnormalities of morphogenesis which indicates insult to early embryologic development (Miles et al. 2005). This group of children were defined as ‘complex autism', while children with no dysmorphic features were classified as 'essential autism’. According to their subtype classification, 20\% of their studied population was classified into the ‘complex autism' subtype and the remainder $80 \%$ of subjects were classified into 'essential autism'. Subjects in the essential group reported a higher heritable recurrence, higher chance of siblings and relatives autism recurrence (Miles and Hillman, 2000). Individuals in the 'complex autism’ subgroup were associated with lower Intelligence Quotients (IQ), experienced more seizures and more abnormal electroencephalography (EEGs).

Myers and Johnson (2007) reviewed the reported prevalence of epilepsy among patients with ASD ranging from $11 \%$ to $39 \%$; and epileptiform abnormalities on EEG, ranging from $10 \%-72 \%$. It was also reported that the percentage of abnormal brain structures shown in Magnetic Resonance Image (MRI) scans are relatively higher in phenotypically abnormal autistic individuals (29\%) than the normal proposition (14\%) (Miles and Hillman, 2000). Study by Hrdlicka et al. (2005) attempted to subtype their autistic subjects into four clusters based on structural MRI data. Subjects in cluster 1 involved the largest sizes of genu and splenium of the corpus callosum; cluster 2 with the largest sizes of the amygdala and hippocampus; 
cluster 3 with the largest size of the head of caudate nucleus, smallest size of the hippocampus; and cluster 4 with the smallest size of the genu of the corpus callosum and splenium of the corpus callosum, as well as the amygdale and head of caudate nucleus. Moreover, cluster 1 shows the lowest frequency of facial dysmorphic features, while facial dysmorphic features are always present in subjects of cluster 3 and 4. The above literature supports the existence of subtypes in ASD based on genetic heterogeneity which may involve abnormalities of early morphogenesis. Based on this premise, we support the approach for subtyping ASD with different fundamental causes.

Currently there is no cure for ASD, and the primary focus for paediatrians and clinical psychologists is the emphasis on the course of treatment through a range of therapies (physiotherapy, counselling, speech, communication and social, etc) with the main goal in mind to improve how ASD patients function in their daily lives. According to Tang et al. (2008), the rate of language and developmental delay are 33\% and 19\% respectively in ASD children diagnosed in Study Country. There are strong evidence to support the existence for a range of variability within ASD children.

With this variability, it is suggested that ASD is comprised of different subgroups genetically and phenotypically. Hence, being able to subcategorize ASD patients into different classifications based on their etiology would aid clinicans to have a more accurate diagnosis and to aid in determining the prognosis of individual ASD patient. We hope with the gain in knowledge in ASD, clinicians can tailor treatment plans for each patient and improve the overall quality of care for patients 
with ASD. In order to find out the heterogeneity within the ASD, biologically-based phenotypes has to be investigated to develop effective treatments (Miles et al. 2008). Although the height, weight and Body Mass Index (BMI) of the autistic children in China has previously been studied (Xiong et al. 2009), up to the time of the present paper, physical abnormalities and dysmorphic features among ASD children in the Chinese population have not yet been studied. Our aim of the current study is to find out the prevalence of presentation of dysmorphic features and to distinguish fundamentally different subgroups in Chinese ASD population.

\section{Methodology}

\section{Design and Study Subjects}

This is a retrospective study reporting on a database of ASD patients from the Study Country at Study Country’s Hospital. The study sample consisted of 1261 Chinese patients diagnosed with Autistic Spectrum Disorder/ Autistic Disorder/ High Functioning Autism/ Asperger’s Syndrome/ Pervasive Developmental Disorder-Not Otherwise Specified (PDD-NOS) by developmental paediatrician, clinical psychologist, and/or clinical neurologist at the Study Country. All patients in the database met the diagnostic criteria of ASD in Diagnostic and Statistical Manual of Mental Disorders, Fourth Edition (DSM-IV: APA,1994).

Patients co-diagnosed with William Syndrome, Tuberous sclerosis, Rett Syndrome, Fragile X Syndrome, Down Syndrome, Dravet Syndrome, Crouzon Syndrome, Sticker Syndrome, Kabuki Syndrome, Angelman Syndrome and Soto Syndrome were excluded. We excluded all subjects with identifiable syndromes to 
prevent confounding factors other than effects of ASD. The data have been pooled from the medical records and examination for dysmorphic features is part of the examination for all children with ASD assessed in our Study Country for medical consultation, thus written consents are not available.

\section{Clinical Evaluation and Morphological Classification}

The current study is a retrospective analysis of case records of the Study Country. Children with neurodevelopmental disabilities assessed in Study Country are collaborated with the University of Study Country on research, training and clinical services especially for ASD (Wong 2008, Wong et al. 2012). A multidisciplinary team approach comprised of a diagnostic team of paediatricians, clinical psychologist, physiotherapist, occupational therapist, speech therapist, optometrists and audiologist. Clinical evaluation comprised of a standard data set for collecting information including prenatal, perinatal, developmental, neurologic, health and family history. A two to four generation pedigree was collected. Social-economical data of the patients' family and medical testing results were reviewed. History of the patients’ autistic symptoms including delay or deficits in language, gross and fine motor, social interactions and behaviors were recorded. Laboratory tests including blood lead and mercury level, urine metabolic screen, thyroid profile, chromosome study, EEG, Computed Tomography (CT), MRI, Positron emission tomography (PET) and Deoxyribonucleic acid (DNA) testing were recorded if otherwise performed. Developmental paediatricians or child neurologists would examine all autistic children during the initial visit for any dysmorphic features as part of the physical examination, and follow-up visits were preformed whenever necessary. 
Physical examinations were performed by pediatricians, developmental paediatricians, child neurologists for dysmorphic features including head, face, hands, feet, body proportions, relative size, position, proportions and shape of each body parts including the cranium, face, eyes, nose, mouth, ears, thorax, back, genitalia, limbs, skin, hair and teeth were evaluated. Recordings of any abnormalities were preformed upon observation. Parents and other relatives were examined if and when available and then recorded in the database. Subjects were defined as dysmorphic (generalized dysmorphology) if physical abnormalities, measurements abnormalities, descriptive features and malformations were observed. Individuals with no physical abnormalities were defined as 'non-dysmorphic'.

\section{Family history}

Family history was obtained via interviews during the consultations. Information including, family socioeconomic status, disease specific questions and family history of autism were obtained. Number of siblings of the patients, history of medical, psychological, behavioral and history of language delay in the family were also recorded. A family history of autism was considered significant when there is an affected first degree relative; or when there is a second degree relative affected and two additional affected individual in the family branch.

\section{Statistical analysis}

Statistical analysis was performed using Statistical Package for Social Sciences (SPSS, version 10.0). For statistical analysis, Chi-square tests were used to compare the frequencies of non-dysmorphic and dysmorphic status within the patients in the ASD database. For categorical random variables, uni-variate analysis was used to 
compare the dysmorphic and non-dysmorphic groups using chi-square tests. Mean age and gender ratio comparison was made using Independent Student $t$-test. Available information on MRI abnormalities, EEGs, epilepsy, IQ and family history was compared using the Independent-student-t-test. A two-tailed $p$ value $<=0.05$ was considered a statistically significant outcome.

\section{Results}

In this study, a Chinese population with 1261 ASD patients whom were evaluated in local hospitals in Study Country between years 1985 - 2010 fulfilling Diagnostic and Statistical manual of Mental Disorders, Fourth Edition (DSM-IV: APA, 1994) criteria for Autism Spectrum Disorder/ Autistic Disorder/ High-functioning Autism/ Asperger's Syndrome/ PDD-NOS were included in the study. The study populations of ASD patients were further categorized based on the presence or absence of minor anomalies, malformations or dysmorphic features. In our study, 136 (10.79\%) patients were classified by our paediatrians as dysmorphic; and 1125 (89.21\%) patients were classified as non-dysmorphic. The mean age of diagnosis for patients are 7.19 years (range of last follow up age, 0-32.78 years) and 6.55 years (range of last follow up age, 0-29.90) for the dysmorphic and non-dysmorphic groups, respectively (Table 1).

Table (2) showed the cross-tabulation of social economical class of the parents with the two groups of patients. A relatively large proportion of the patients came from families of higher social economical status. As some of the parents of the 
patients chose not reveal their economical status, the total number of parents indicated in Table (2) did not add up to 1261.

Phenotypic features were considered to be useful in separating autism into more homogeneous subgroups other than behavioral diagnosis according to Miles et al. (2005). Malformations are originated from first trimester of pregnancy and hence, dysmorphology was identified as one of the most informative measures since they are present from birth. Presence of dysmorphic features provide clues which may suggest a specific subgroup of the autistic individuals may have underwent insults to their early embryological development. Descriptions of any dysmorphic features observed on the patients were recorded descriptively and the number of times of the features sited in the study was counted. Among all the features identified in the dysmorphic subject group, macrocephaly, prominent forehead, big ears and hypertelorism showed highest occurrence (Table 3).

Brain MRIs were obtained for 186 (14.75\%) patients. (Note: MRIs were not part of a standardized assessment for children with neurological disorders, and hence not all our study population obtained MRI reports). The MRIs were performed in hospitals and under strict radiographic protocols. All MRI results were reviewed by experienced neuro-radiologist uninformed of the hypothesis of the autism sub categorization or phenotypic status.

Intellectual quotient (IQ) or Developmental quotient (DQ) assessments were available on 723 (57.34\%) patients. Patients were evaluated by developmental pediatricians and/or clinical psychologist in the team. Intellectual ability was assessed 
by Griffiths Mental Developmental Test (Griffiths 1970) for evaluation of DQ. Patients were subdivided into different groups according to the learning disability (LD) and IQ scores, including normal, limited LD, mild LD, moderate LD and severe LD. IQ scores comparison between the dysmorphic and non-dysmorphic groups was performed with the 723 individuals with available scores (Table 4).

Gender and EEG results showed no significant differences between the two groups, while uni-variate analysis showed that epilepsy, IQ scores, MRI/CT result and family history of language delay were significant risk factors for dysmorphic $(\mathrm{p}<0.05)$ (Table 4, 5).

Table 3 showed the number of times a dysmorphic feature was cited in the database. Any dysmorphic features noted by medical examiners were recorded descriptively upon physical examination based on their clinical impression. For the purpose of this study, the number of times of a dysmorphic feature noted was counted. Among all the dysmorphic features - marcocephaly, frontal bossing of the forehead, prominent ears yielded a relatively higher occurrence frequency. Macrocephaly was noted in $27.94 \%$ of the dysmorphic patients, $20.59 \%$ presented with frontal bossing of the forehead while $24.26 \%$ presented with prominent ears. Other informative dysmorpholgy designations include hypertelorism, flat nasal bridge and clindodactyly which occur in $11.76 \%, 8.09 \%$ and $5.88 \%$ respectively.

Our results show that phenotypic status positively correlates with abnormal structures of the brain. In the dysmorphic group, $40.5 \%$ of the patients showed abnormal brain structure while $24.2 \%$ in the non-dysmorphic group had abnormal 
structures with $p=0.046$ (Table 4). Brain abnormalities included abnormalities affecting the cerebellum and the cerebrum including the frontal, temporal, parietal, occipital area, asymmetry difference of the cerebrum, hydrocephalus, delayed myelination, occurrence of cysts and atrophy of the cerebrum and/or cerebellum.

In our study, $14 \%$ of the patients with dysmorphic features exhibited epilepsy while only $4.8 \%$ of the patients without dysmorphic feature exhibited epilepsy (Table 4). Significant difference was found with a $p$-value of 0.000 , highly suggestive that seizures is an important risk factor for a dysmorphic sub categorization.

Comparing LD and IQ scores, significant differences were noted in the mild and moderate LD groups with $p$ value of 0.001 and 0.009 respectively (Table 4). No significant differences were found between the limited IQ and severely delayed groups. In the non-dysmorphic group, $40.8 \%$ had normal or limited IQ where the dysmorphic group only comprised of $20 \%$. Higher percentage of patients with mild to moderate LD were found in the dysmorphic groups with $43.8 \%$ as mild and $31.4 \%$ as moderate, while only $29.6 \%$ and $25.7 \%$ were diagnosed as mild and moderate LD respectively in the non-dysmorphic group.

In this study, we did not find a significant difference in sex ratio and family history of autism in the two groups; and abnormal EEG results were not significant in the dysmorphic group.

\section{Discussion}


Investigation on specific dysmorphology features was useful in identifying many genetic disorders or syndromes. Currently, there is an increased interest in the investigation of physical phenotypic features in autism in recent researches trying to separate autism population into genetic subsets for investigations. There are previous studies for the Caucasian population which shows there are phenotypic subgroups of autism by observing their minor physical anomalies and dysmorphic features (Rodier et al. 1997; Fombonne et al. 1999; Miles et al. 2008; Tripi et al. 2008). Group differences were found between dysmorphic and non-dysmorphic patients, showing that with any early insult to morphogenesis is generally etiologically different from those of normal development. While genetics have an important role in the development of autism, it is difficult to identify autism causing genes, so genetically predictive phenotypic markers has become an interest of investigation. Dysmorphology studies picks up clues in identifying disturbances in embryological development or mutations of genes which originates during pregnancy. Early disturbance in embryonic development could not be altered by subsequence illness and some specific anomalies shown seemed to have a high prevalence with the disorder.

In the current study, the highest rate of dysmorphic features is associated to the cranium area, with a high rate of macrocephaly and frontal bossing. This finding coincides well with previous Caucasian studies (Rodier et al. 1997; Fombonne et al. 1999; Miles et al. 2008; Tripi et al. 2008). It has also been suggested that macrocephaly in children with autism is related to increase in total brain volume. Nelson and Nelson (2005) concluded that around 20-30\% of the autistics patients had head sizes more than 2 standard deviations with the normal comparison group, and 
MRI assessment show general increased cerebral volume. Deutsch and Joseph (2003) found that increased head circumference correlates with non verbal IQ scores, suggesting reflection of disturbance in neurocognitive development and organization of the brain in some autistic patients, proposing a possible etiologically significant subtype of autism.

The result of this study agrees fairly well with an earlier study by Miles et al. (2005), reporting the highest occurrences of abnormalities found in nose structure and size, ear size and cranial shape and size. Higher frequency of hypertelorism was noted as dysmorphic features in Chinese population compared to the Caucasians and high percentage of clindodactyly agrees with the study of Tripi et al. (2008). However, we did not find deep-set nails, tapering fingers, low anterior hairline and thin upper lip as commonly observed in our dysmorphic autism group as in the Caucasian population.

In our study, the occurrence of prominent ears, including size, structure and/or orientation of ear in children with autism was relatively high; as similarly reported in other studies by Rodier et al. (1997). Their study found posterior rotation of ear as an important indicator in distinguishing autistic patients with controls while in our current study, big prominent ears was shown to be one of the most highly identified features. With a high occurrence rate, genetically caused autism might suggest accompaniment of ear abnormalities during development. However information on ear development, including the development sequence of the inner and outer ear structures and their interaction during prenatal period is not clear, and more investigation is required towards this finding. 
Other more frequently identified features in this study included hypertelorism, flat nasal bridge and clinodactyly. Hypertelorism yielded a relatively high occurrence in our Chinese autism group, a finding conflicting with the study of Rodier et al. (1997) in which they found that short interpupillary distance was significant in Caucasian autism group. Moreover, dysmorphic features related to eye structures and position were less reported in the Caucasian population, though deep set eyes were reported as one of the autism related features in the study by Miles et al. (2005). However, our findings on flat nasal bridge and clinodactyly agree with previous studies in which these features are related with midline brain anomalies, chromosome abnormalities and autism.

The findings in our study generally agrees with the results of the Caucasian population which patients with dysmorphic features presents with a higher incidence of abnormal brain structure, lower IQ level and higher epilepsy rate. This supports the study by Miles et al., in which classification of autism subtypes by dysmorphic features into the subcategories of ‘essential autism' and ‘complex autism'. Miles et al. (2005) further suggested that patients with complex autism is less heritable and more apt to be due to sporadic mutation, including point mutation, deletion, insertions, duplication and other chromosomal rearrangements, which were shown to be more dysmorphic in physical appearances. Though we did not found a significant difference in sex ratio between both groups, result in the current study did show significant correlation of dysmorphology with abnormal brain structure, IQ and seizure rate. This also supports findings in previous Caucasian studies suggesting that a heterogeneous ASD group originates from mutation of certain embryological important genes, leading to anomalies in the development of the brain and face. However, due to 
limitation of resources, number of MRI and IQ test were unavailable for all ASD patients in our study group, future studies might consider comparing with a larger sample size.

As this study took a retrospective approach, a major limitation is that all of the data were collected were case-note based and serve to report the on-going medical information for ASD patients. The classification of dysmorphology was based entirely on examiner’s judgement in physical examination of generalized dysmorphology. Any physical anomaly that was noted was reported by the examiner and then classified into (non)dysmorphological status. This retrospective study lacks a standard scale for measuring and defining dysmorphology, and thus, our study group have initiated an on-going prospective study with a unify measure for classifying dysmorphology status within the Chinese population.

It was suggested that phenotypically different autistic patients have different prognosis and predictive outcomes. In study by Miles et al. (2005), individuals with 'complex autism', presented with dysmorphic features perform less well in all of their outcome measures, including their IQ scores and verbal ability after 8 years of age. In our current study, it is shown that patients with dysmorphic features presented with poorer IQ scores. In our study, language ability was not measured, and therefore verbal ability could not be compared. However, interestingly, factor of language delay in family history showed significant result in distinguishing the dysmorphic and non-dysmorphic groups $(\mathrm{p}=0.023)$. Language ability is another genetically inherited factor and it is highly correlated with cognitive level. A main diagnostic criteria for ASD is uneven language development, while many children with ASD do develop 
some speech and language skills, their development is often not to a normal level of ability, but of uneven progress. Genome studies were performed in relating specific genes with different language problems. Localization of genes related with language impairments were performed in numerous studies. Reviews done by Grigorenko (2009) and Smith (2007) summarized the relationship of specific genes with speech and language problems associated with autism, dyslexia and specific language impairment. Dworzynski et al. (2007) also found modest correlation with low language performance with later autistic-like traits. This suggested that language ability is highly genetically dependent. Therefore, in future genetically based autism studies, language and verbal ability are key areas of future research interest.

The results observed in the current investigation appear to be fairly consistent with previous studies with the Caucasian population. The diversity of result found in both Caucasian and Chinese population suggests a morphological heterogeneity of autistic spectrum disorder and its wide clinical phenotype with different most informative dysmorphology designations found in different subject groups and ethnical population.

\section{Conclusion}

To conclude, data gained from the Chinese ASD population generally resembled result shown in previous Caucasian studies. However, ethnical differences were still present and it is important to set up a norm database of different body measurements of the Chinese population in order to compare data of the ASD patients to the normal standard for finding out their standard deviation for more precise investigations (Wong 2012). Furthermore, training of clinicians for differentiating 
dysmorphic and non-dysmorphic patients might allow better diagnostic classification of their autistic subjects, to help determining their prognosis and for more effective referrals to different treatments, laboratory assessments and decisions of referrals for schooling. 


\section{References}

Autism and Developmental Disabilities Monitoring Network (ADDM) (2012).

"Prevalence of autism spectrum disorders--Autism and Developmental Disabilities Monitoring Network, 14 sites, United States, 2008.” MMWR Surveill Summ; 61(3): p1-19.

Allanson, J.E., Bieseckel, L.G., Carey, J.C. and Hennekam, R.C.M. (2008). “Elements of Morphology: Introduction.” American Journal of Medical Genetics: p2-5.

Allanson, J.E., Cunniff, C,. Hoyme, H.E., McGaughran, J., Muenke, M., and Neri, G.(2009). "Elements of Morphology: Standard Terminology for the Head and Face.” American Journal of Medical Genetics: p6-28.

American Psychiatric Association (1994). Diagnostic and statistical manual of mental disorders ( $4^{\text {th }}$ ed.). Washington, DC.

Bauman, M.L., and Kemper, T.L. (2004). Neurobiology of Autism. The Johns Hopkins University Press. Baltimore, M.D. USA.

Biesecker, L.G., Aase, J.M., Clericuzio, C., Gurrieri, F., Temple, I.K., and Toriello, H. (2009). "Elements of Morphology: Standard Terminology for the Hands and Feet." American Journal of Medical Genetics Part A : p93-127.

Carey, J.C. (2008). “Editorial Comment: Editor’s Foreword to a Special Issue 'Elements of Morphology: Standard Terminology.” American Journal of Medical Genetics: p1.

Carey J.C., Cohen Jr, M.M., Curry, C.J.R., Devriendt, K., Holmes, L.B., and Verloes, A. (2009). "Elements of Morphology: Standard Terminology for the Lips, Mouth, and Oral Region.” American Journal of Medical Genetics: p77-92.

Census and Statistics Department in Hong Kong (2008). "Special Topics Report No. 48 - Persons with disabilities and chronic diseases". Retrieved August 102010 from, http://gia.info.gov.hk/general/200812/22/P200812220123_0123_48059.pdf 
Deutsch, C.K. and Joseph, R.M. (2003). "Brief Report: Cognitive Correlates of Enlarged Head Circumference in Children with Autism.” Journal of Autism and Developmental Disorders. 33: p209-215.

Dworzynski, K., Ronald, A., Hayiou-Thomas, M., Rijsdijk, F., Happe, F., Bolton, P. F., \& Plomin, R. (2007). “Aetiological relationship between language performance and autistic-like traits in childhood: a twin study”. Int J Lang Commun Disord, 42(3): p273-292.

Fombonne, E., Roge, B., Claverie, J., Courty, S., and Fremolle, J. (1999).

"Microcephaly and Macrocephaly in Autism.” Journal of Autism and Developmental Disorder. 29: p113-119.

Grigorenko, E. L. (2009). "Speaking genes or genes for speaking? Deciphering the genetics of speech and language.” J Child Psychol Psychiatry, 50(1-2): p116-125.

Griffiths, R. (1970). The Abilities of Young Children: A Comprehensive System of Mental Measurement for the First Eight Years of Life. Test Agency Ltd. North Dean, United Kingdom.

Hall, B.D., Graham Jr, J.M., Cassidy, S.B., and Opitz, J.M. (2009). "Elements of Morphology: Standard Terminology for the Periorbital Region.” American Journal of Medical Genetics: p29-39.

Hennrkam, R.C.M., Cormier-Daire, V., Hall, J.G., Mehes, K., Patton, M., and Stevenson, R.E. (2009). "Elements of Morphology: Standard Terminology for the Nose and Philtrum.” American Journal of Medical Genetics Part A: p61-76.

Hunter, A., Frias, J.L., Gillesse-Kaesbach, G., Hughes, H., Jones, K.L., and Wilson, L. (2009). "Elements of Morphology: Standard Terminology of the Ear.” American Journal of Medical Genetics Part A: p40-60.

Hrdlicka, M., Dudova, I., Beranova, I., Lisy, J., Belson, T., Neuwirth, J., Komarek, V., Faladova, L., Havlovicova, M., Sedlacek, Z., Blatny, M., and Urbanek, T. (2005). "Subtypes of Autism by Cluster Analysis Based on Structural MRI Data.” European Child \& Adolescent Psychiatry. 14: p138-144. 
Kogan, M.D., Blumberg, S.J., Schieve, L.A., Boyle, C.A., Perrin, J.M., Ghandour, R.M., Singh, G.K., Strickland, B.B., Trevathan, E., and C. van Dyck, P. (2009). "Prevalence of Parent-Reported Diagnosis of Autism Spectrum Disorder Among Children in the US, 2007.” Pediatrics; 124: p1395 - 1403.

Lord, C., and Rutter, M. (1994). “Autism diagnostic interview-revised: a revised version of a diagnostic interview for caregivers of individuals with possible pervasive developmental disorders.” Journal of Autism and Developmental Disorders. 24(5): p659-685.

Lord, C., and Rutter, M. (1989). “Autism diagnostic observation schedule: a standardized observation of communicative and social behavior.”Journal of Autism and Developmental Disorders, 19(2): p185-212.

Miles, J.H., and Hillman, R.E. (2000). "Value of a Clinical Morphology Examination in Autism.” American Journal of Medical Genetics. 91: p245-253.

Miles, J.H., Takahashi, T.N., Bagby, S., Sahota, P.K., Vaslow, D.F., Wang, C.H., and Hillman, R.E. (2005). "Essential Versus Complex Autism: Definition of Fundamental Prognostic Subtypes.”American Journal of Medical Genetics; 135A: p171-180

Miles, J.H., Takahashi, T.N., Hong J., Munden, N., Flournoy, N., Braddock, S.R., Martin, R.A., Spence, M.A., Hillman, R.E. and Farmer, J.E. (2008). “Developmental and Validation of a Measure of Dysmorphology: Useful for Autism Subgroup Classification.” American Journal of Medical Genetics Part A; 146A: p1101-1116

Miles, J.H. (2008). “Autism Dysmorphology Measure (ADM) Training Manual.” Supplement, University of Missouri.

Nelson, K.B. and Nelson, P.G. (2005). "Size of the head and brain in autism: Clue to underlying biologic mechanisms?” In M. Bauman \& T. Kemper (Eds.) The neurobiology of autism, 2nd ed., (p23-33). Baltimore: Johns Hopkins University Press.

Ozgen, H.M., Hop, J.W., Hox, J.J., Beemer, F.A., and Engleland, H.V. (2010). “Minor Physical Anomalies in Autism: A Meta-analysis.” Molecular Psychiatry. 15: p300-307. 
Rodier, P.M., Bryson, S.E., and Welch, J.P. (1997). "Minor Malformations and Physical Measurements in Autism: Data from Nova Scotia.” Teratology; 55: p319-325.

Myers, M.S., and Johnson, C.P.J. (2007). "Management of Children with Autism Spectrum Disorders.” Pediatrics. 120: p1162-1182.

Smith, S. D. (2007). “Genes, language development, and language disorders.” Ment Retard Dev Disabil Res Rev, 13(1): p96-105.

Tang, M.L., Chen, K.Y., Lau, W.Y., and Wu, M.F. (2008). “Clinical Profile of Young Children with Mental Retardation and Developmental Delay in Hong Kong.” Hong Kong Medical Journal. 14: p97-102.

Tripi, G., Roux, S., Canziani, T., Brilhault, F.B., Barthelemy, C., and Canziani, F. (2008). "Minor Physical Anomalies in Children with Autism Spectrum Disorder.“ Early Human Development. 84: p217-223.

Wechsler, D. (1989). Wechsler Preschool and Primary Scale of Intelligence-Revised. San Antonio: The Psychological Corporation

World Health Organization (WHO). (2007). ICD-10 international statistical classification of diseases and related health problems. Geneva, World Health Organization.

Wong, P.T.Y. (2012). "Pilot study for subgroup classification for Autism Spectrum Disorder based on dysorphology and physical measurements in Chinese children.” Dissertation. The University of Hong Kong, Retrieved from February 21, 2012, from Hong Kong University thesis Online HKUTO, http://sunzil.lib.hku.hk/hkuto/index.jsp.

Wong, V., Hui, S., Lee, W.C., Leung, L.S., Ho, P.K, Lau, W.L. Fung, C.W., and Chung, B. (2004). “A Modified Screening Tool for Autism (Checklist for Autism in Toddlers [CHAT-23]) for Chinese Children.” Pediatrics; 114: p166-176.

Wong, V., and Hui, S. (2008). "Brief Report: Emerging Services for Children with Autism Spectrum Disorders in Hong Kong (1960-2004).” Journal of Autism and Developmental Disorders, 38: p383-389. 
Wong. V., and Hui, S. (2008). "Epidermiological Study of Autism Spectrum Disorder in China.” Journal of Child Neurology, 23: p67-72.

Wong, V.C.N., Fung, C.W., Lee, S. L., and Wong, P.T.Y. (2012). "Review of evolution of clinical, training and educational services and research program for autism spectrum disorders in Hong Kong.” Science China Life Science, 55: p1-18.

Wu, K.H., Tsai, F.J., Li, T.C., Tsai, C.H., Peng, C.T., and Wang T.R. (2000).

"Normal Values of Inner Canthal Distance, Interpupillary Distance and Palpebral Fissure Length in Normal Chinese Children in Taiwan.” Acta Paediatric Tw. 41: p22-27.

Xiong, N., Ji, C.Y., Li, Y. He, Z.H., Bo, H.L., and Zhao, Y.F. (2009). “The Physical Status of Children with autism in China.” Research in Developmental Disabilities, 30: p70-76. 